Albert Wettstein ${ }^{1}$, Matthias Peters ${ }^{2}$

1 Stadtärztlicher Dienst, Zürich

${ }^{2}$ IPSO Sozialforschung, Dübendorf

\section{Die Tragfähigkeit der sozialen Netze von Einpersonenhaushalten bei akuter Krankheit}

\author{
Basisdaten zur Spitexplanung bei Grippepandemie
}

haben, mit dem sie wichtige Entscheide besprechen können ${ }^{2}$.

Analog wurde in der Berliner Altersstudie ${ }^{3}$ dokumentiert, dass $87 \%$ der untersuchten Betagten Hilfe von Mitgliedern ihres sozialen Netzes empfangen und $86 \%$ aktive Hilfe an Netzwerkpartner leisten. In beiden Studien ${ }^{2,3}$ ist jedoch emotionale Unterstützung ein wesentlicher Teil der Hilfsbeziehungen und diese wiederum sind nur eine Teilmenge der Beziehungen im sozialen Netz.

Gemäss Barnes ${ }^{4}$ und Mitchell ${ }^{5}$ ist das soziale Netzwerk gemäss dem Inhalt der sozialen Beziehungen aufzuteilen in die Bereiche Information und Kommunikation, ferner Austausch und Transaktion sowie Übermittlung von Normen, Werten und Attitüden, wobei die einzelnen Beziehungen uniplex oder multiplex sein können, je nach der Anzahl Inhalte. Aus der SIMA VIII Studie ${ }^{6}$ von Betagten in Erlangen ist Näheres darüber bekannt: Alle 276 Teilnehmer haben mindestens einen Netzwerkpartner, durchschnittlich $8,5 \pm 3,5$ (nur 1,5\% haben nur einen Partner, aber $2,5 \%$ mehr als 16 Partner). Aber $28 \%$ haben keinen Netzwerkpartner für ein persönliches Gespräch, $36 \%$ niemand zur Hilfe bei der Entscheidungssuche, $47 \%$ keine Partner, denen sie sel- 
ber Hilfe leisten, sowie $33 \%$ niemanden, von dem sie Hilfe empfangen haben (in den letzten drei Monaten). $75 \%$ der Netzwerkbeziehungen spielen keine Rolle für das Geben oder Annehmen von Hilfe, welche zu je einem Drittel seltener als einmal monatlich, mehrmals pro Monat sowie mehrmals wöchentlich oder täglich erfolgt.

Hilfeleistungen werden vor allem von nahen Verwandten $(17 \%)$, Nachbarn $(18 \%)$ und weniger von Freunden und Bekannten (4\%) erbracht. Die Akzeptanz von Hilfeleistungen ist bei $24 \%$ der Befragten gering und bei $32 \%$ hoch. Dabei ist eine hohe Akzeptanz von Hilfe vor allem bei Befragten anzutreffen, die früher selber Hilfe geleistet haben oder die Helfenden das Gefühl vermitteln, gebraucht zu werden.

Während die Sozialnetzwerke der Betagten und dabei insbesondere die Unterstützungsnetze recht gut untersucht sind, bestehen für die ganze grossstädtische Bevölkerung weniger klare Verhältnisse. Der kulturpessimistischen Sicht der "anonymen Massengesellschaft" entspricht die Überzeugung, die feste Einbindung in Verwandtschaftssysteme und lokale Nachbarschaften seien durch veränderte Bedingungen der Umwelt und der Nachbarschaft weitgehend gelockert, so dass eine Integration in soziale Netzwerke nicht möglich sei $^{7}$. Dem gegenüber steht die Überzeugung, dass neben die früher weitgehend vorgegebenen sozialen Netze zunehmend solche sozialen Bindungen treten, die auf freiwilliger Wahl der Betreffenden beruhen und die dichten multiplexen Beziehungsgeflechte traditioneller Sozialstrukturen ersetzen $^{8}$. Empirische Untersuchungen $^{9,10}$ belegen jedoch, dass von einer völligen Verdrängung von Primärbeziehungen nicht die Rede sein kann und enge Bindungen im Verwandtschafts- und Freundeskreis sowie zu Nachbarn und Ar- beitskollegen auch in modernen Grossstädten nachweisbar sind.

Die Frage nach Austauschbeziehungen in den persönlichen Netzwerken ist ebenfalls für Hilfsbedürftige jeden Alters dokumentiert $^{11}$. Dabei stellt sich heraus, dass Nachbarn eher Hilfe in alltäglichen Dingen leisten, während in schwierigen Angelegenheiten die geografische Nähe der Netzwerkpartner unwichtig ist.

Die Datenlage ist jedoch ungenügend, um zuverlässig abschätzen zu können, ob auch in einer grossen Schweizer Stadt wie Zürich die sozialen Netzwerke der $\mathrm{EPH}$ in der Lage sind, in sozialmedizinisch relevantem Ausmass angemessen Hilfe zu leisten, z.B. bei einer Grippepandemie, $\mathrm{zu}$ der es in den kommenden Jahren wahrscheinlich einmal kommen wird $^{12-14}$.

Im schlimmsten Fall muss dabei wie 1918 in Philadelphia ${ }^{15}$ - mit einem Befall von $50 \%$ der Bevölkerung innert sechs Wochen gerechnet werden. In einer solchen Situation ist auf dem Gipfel der Epidemiewelle - in der Woche mit den meisten Erkrankungen - ein Sechstel der Bevölkerung bettlägerig und auf Hilfe angewiesen. Da nur mit einer hospitalisierungsbedingenden Komplikationsrate von max. $2 \%$ zu rechnen ist ${ }^{15}$, wird das gut ausgebaute städtische Spitalwesen dies bewältigen können (max. 1200 Hospitalisationen pro Woche bei einem Angebot von über 2000 Spitalbetten für Einwohner der Stadt Zürich).

Unklar ist hingegen, wie stark das spitalexterne System für einen solchen Fall auszubauen ist, für wieviele Kranke in EPH die Öffentlichkeit Hilfe bereitstellen muss, weil das persönliche soziale Netz nicht genügt. Um die Planung auf Fakten, nicht nur auf Vermutungen abstellen zu können, wurde eine Befragung von EPH in der Stadt Zürich durchgeführt, mit dem Ziel festzustellen, für wieviele dieser Haushalte das soziale Netz im Falle einer kurzen akuten Krankheit ungenügend ist.

\section{Methodik}

Die vom Einwohneramt der Stadt geführte Erfassung der EPH ist unzuverlässig und schliesst ca. einen Drittel unechter EPH mit ein, d.h. Wohnungen, in denen mehrere Personen zusammen wohnen, sei es in Untermiete $(6,9 \%$ gemäss Volkszählung $\left.1990^{1}\right)$ oder in Wohngemeinschaften (Anzahl unbekannt). Deshalb wäre mit prohibitivem Mehraufwand verbunden gewesen, wie ursprünglich vorgesehen, eine Zufallsstichprobe der registrierten EPH der Stadt Zürich zu untersuchen. Es wurden deshalb zwei nicht zufällig ausgewählte Populationen von EPH untersucht:

1. Anlässlich aller von April bis Juli 1998 stattfindenden Rapporte zur Einteilung der neu zivilschutzpflichtig gewordenen Einwohner der Stadt Zürich wurden die Teilnehmer vom leitenden Instruktor befragt, ob sie alleine lebten. Bejahende wurden gebeten, einen Fragebogen auszufüllen. Ein von den Autoren geschulter Instruktor leitete die befragten Personen an. $100 \%$ der so erfassten $136 \mathrm{EPH}$ nahmen an der Studie teil.

2. 12 Aktive Samariter aus den Zürcher Stadtkreise 6, 10, 11 und 12 erklärten sich bereit, je mehrere Personen in EPH persönlich zu befragen. Sie sollten Interviews durchführen mit möglichst ähnlich vielen $\mathrm{Be}$ kannten und Nachbarn, von denen sie annahmen, sie seien isoliert, als auch mit solchen, die sie als nicht-isoliert einschätzten. Von den vermutlich nicht Isolierten beteiligten sich $95 \%$, (77 Personen), von den isoliert Lebenden $80 \%$ (17 Personen) an der Befragung. Insgesamt wurden so 103 Personen kon- 


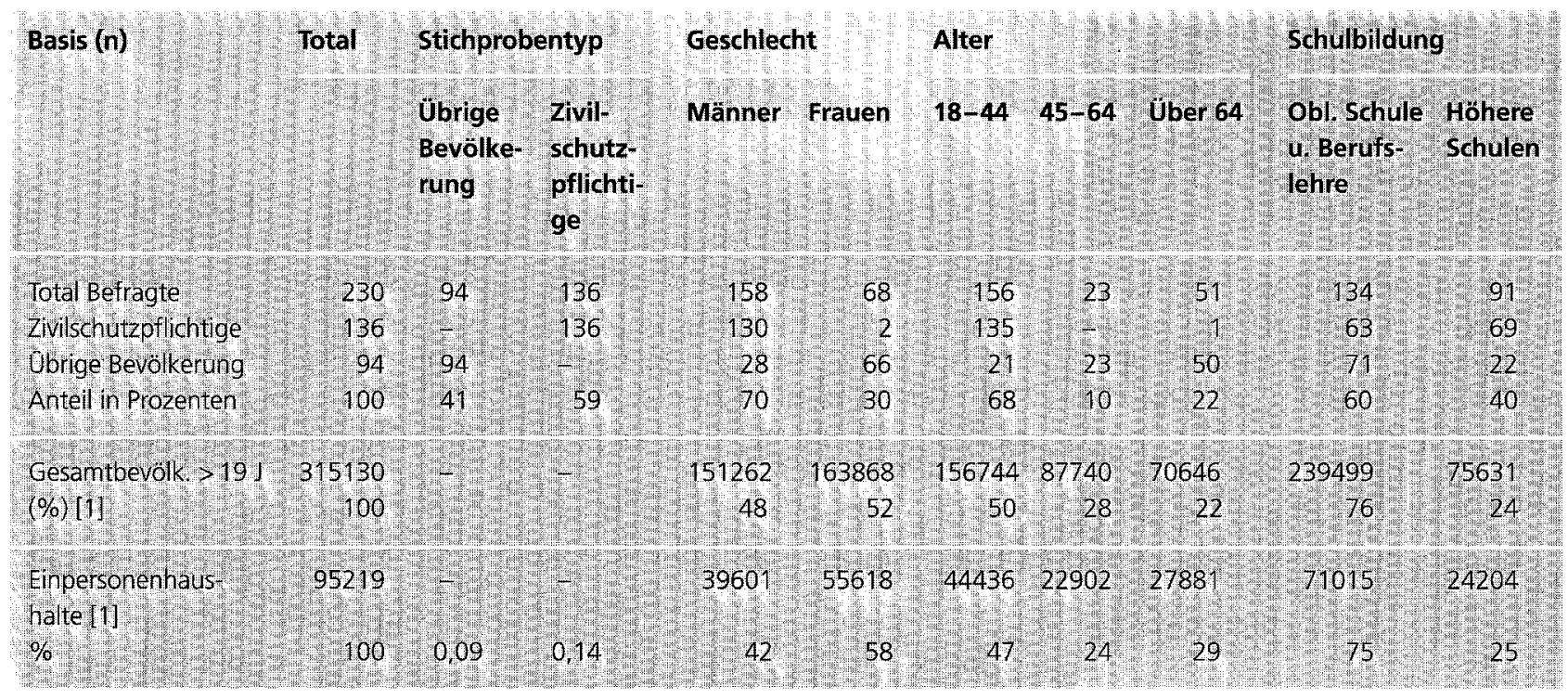

Tabelle 1. Zahlenbasis der Untersuchung.

taktiert, wovon $91 \%$ (94 Personen) befragt werden konnten.

Die sozioökonomischen Charakteristika der Befragten sind in Tabelle 1 dargestellt.

Insgesamt wurden Bewohner aus allen Stadtquartieren befragt, jedoch nur vereinzelte Personen aus den Quartieren Balgrist, Fluntern und Witikon (Kreis 7). Die nichtzufällige Auswahl der Befragten ergab deutliche Abweichungen von der Gesamtheit der EPH: Frauen: $30 \%$ statt $58 \%$, Junge unter 44 Jahre alt: $68 \%$ statt $47 \%$. Betagte über $64: 22 \%$ statt $29 \%$ und höher Gebildete: $40 \%$ statt $24 \%$. Deshalb wurden alle Fragen sowohl für das gesamte Sample und vier Subsamples ausgewertet:

- die beiden Untersuchungspopulationen Zivilschutzpflichtige und übrige Bevölkerung

- die beiden Geschlechter

- drei Alterskategorien 18-44 Jahre, 45-64 Jahre und über $65 \mathrm{Jah}$ re, sowie

- zwei Bildungsstand-Kategorien: obligatorische Schule/Berufslehre und höhere Schulen.

Mittels Chi-Quadrat-Test wurde untersucht, ob sich die Befunde der
Subsamples signifikant $(\mathrm{p}<0,05)$ unterscheiden; mit „S.S.n.s." werden Ergebnisse bezeichnet, die sich in den vier Subsample-Analysen nicht signifikant unterscheiden. Wenn Mittelwerte angegeben werden, ist in Klammer jeweils mit positiven und negativen Vorzeichen die Standardabweichung eingefügt.

Bei der Interpretation der Daten ist bei Hochrechnungen von einer Stichprobe auf die Grundgesamtheit immer eine Bandbreite (Konfidenzintervall) zu berücksichtigen.

\section{Resultate}

\section{Wohnsituation}

Zur Abschätzung der Anzahl Nachbarn im gleichen Haus wurde die Anzahl Wohnungen im Haus der Befragten erfasst.

Die 230 Befragten leben in Häusern mit durchschnittlich $12( \pm 14)$ Wohnungen, $11(5 \%)$ in Einfamilienhäusern, $27(12 \%)$ in Häusern mit zwei bis fünf Wohnungen, 111 $(48 \%)$ in Häusern mit 6-10 Wohnungen) und $77(31 \%)$ in Häusern mit mehr als 10 Wohnungen. Die Wohnungen enthielten durch- schnittlich 2,4 Zimmer ( $\pm 1,1)$, nämlich $44(19 \%)$ je ein Zimmer, 83 $(36 \%)$ zwei Zimmer, 74 (32\%) drei Zimmer und $22(9 \%)$ vier und mehr Zimmer. In der Volkszählung 1990 wiesen die EPH zu $25 \%$ ein Zimmer, 31\% 2 Zimmer, 30\% 3 Zimmer und $14 \%$ vier und mehr Zimmer auf. Der Unterschied zur untersuchten Stichprobe ist nicht signifikant im Chi-Quadrat-Test.

Die Befragten leben seit durchschnittlich 8,5 Jahren $( \pm 9,5)$ allein und seit durchschnittlich $11,3 \mathrm{Jah}$ ren $( \pm 16,1)$ in der selben Wohnung.

Betagte leben signifikant länger allein nämlich $15,3( \pm 12,3)$, auch Frauen $(16,4( \pm 12,9)$ Jahre $)$ und länger in der gleichen Wohnung, nämlich $27,7 \quad( \pm 18,6)$ resp. 24,4 $( \pm 18,7)$ Jahre. Männer bewohnten signifikant häufiger nur maximal ein Jahr die selbe Wohnung, nämlich $42 \%$ im Vergleich zu $6 \%$ der Frauen.

Nur 8 der 230 Befragten (3,5\%) haben kein Telefon in der eigenen Wohnung.

\section{Familiensituation}

Von den Befragten haben $60 \%$ lebende Eltern, $75 \%$ Geschwister, 
$32 \%$ Kinder, $54 \%$ andere Verwandte und nur 10 Personen $(4,3 \%)$ keine lebenden Familienmitglieder. Durchschnittlich sehen sie regelmässig $1,7( \pm 2,5)$ Familienmitglieder, aber 73 (32\%) sehen Familienmitglieder nicht regelmässig. Dabei fanden sich keine signifikanten Unterschiede in den Subsamples (S.S.n.s.). Die Häufigkeit der Familienkontakt beträgt durchschnittlich 4,4 $( \pm 10,3)$ Kontakte pro Woche (S.S.n.s.). Bei $156(68 \%)$ der Befragten lebten keine Familienangehörigen in der Nachbarschaft $\quad(=1 \mathrm{~km}$ Distanz oder max. $1 / 4$ Std. zu Fuss entfernt).

\section{Übriges soziales Netz}

Die Befragten sehen durchschnittlich 6,6 $( \pm 9,4)$ Freunde und Bekannte regelmässig $1-2 \times$ pro Woche. 13 Personen $(6 \%)$ sehen keine Freunde und Bekannten regelmässig. Durchschnittlich leben 2,1 $( \pm 3)$ Freunde oder Bekannte in der Nachbarschaft, aber bei 79 Personen (34\%) niemand. Insgesamt besteht das soziale Netz (inkl. Familienmitglieder, Freunde und Bekannte), durchschnittlich aus 8,3 Personen, was der Netzgrösse der SIMA VIII Studie ${ }^{6}$ mit 8,5 Personen entspricht.

\section{Befinden}

Häufig einsam fühlen sich $8(3,5 \%)$, $65(28 \%)$ manchmal, $82(36 \%)$ selten und $73(32 \%)$ nie. Das Subsample Betagte fühlt sich signifikant öfters häufig einsam $(12 \%)$, bzw. nie einsam $(47 \%)$. Dies entspricht dem Befund aus dem Tessin, wo sich ebenfalls $12 \%$ der Befragten einsam fühlten ${ }^{16}$.

Die grosse Mehrheit fühlt sich gesundheitlich sehr gut $(30 \%)$ oder gut $(52 \%)$. Nur $37(16 \%)$ fühlen sich gesundheitlich mittelmässig und gar nur zwei Personen schlecht, niemand fühlt sich gesundheitlich sehr schlecht. Das Subsample der Betagten fühlt sich signifikant häufiger, nämlich zu $33 \%$, als nur mittelmässig gesund, zu $2 \%$ als schlecht und nur zu $65 \%$ gut oder sehr gut. Die subjektive Gesundheitseinschätzung der betagten Zürcher ist etwas besser als die selbsteingeschätzte Gesundheit in Genf, wo 1994 über-65jährige diese zu $56 \%$ als gut bis sehr gut, zu $37 \%$ als zufriedenstellend und $\mathrm{zu}$ $7 \%$ als schlecht bezeichneten ${ }^{17}$. Der Unterschied ist gering, und nicht signifikant im t-Test und im Mann-Whitney-U-Test.

Nur $6(2,6 \%)$ nehmen zum Befragungszeitpunkt Spitexhilfe in Anspruch.

\section{Medienkonsum}

Von den Befragten lesen 112 (49\%) fast täglich das Tagblatt, $90(39 \%)$ den Tages-Anzeiger, 33 (14\%) die NZZ, 114 (50\%) hören fast täglich Lokalradio und 82 (36\%) sehen fast täglich Regionalfernsehen. Niemand hat keinen Medienkonsum. Das am weitesten verbreitete Medium, das Tagblatt, wird von $43(19 \%)$ nie und von $30(13 \%)$ nur ein- bis zweimal pro Monat gelesen. Das heisst, dass auch Personen in EPH vollständig erreicht werden können mit einer Medienkampagne, die sich der grossen Tageszeitungen und lokalen Fernseh- und Radiostationen bedient, dass aber blosse Mitteilungen im städtischen Amtsblatt (Tagblatt) dazu nicht genügen. Wie wichtig dies bei gehäuften akuten Erkrankungen ist, ergibt sich daraus, dass 115 (50\%) der Befragten nicht wissen, wie man Spitex anfordern kann (bei Männern, Personen unter 44 Jahren und Höhergebildeten ist der Anteil noch höher, nämlich $65 \%, 64 \%$, resp. $60 \%$ ).

\section{Haushalthilfe-Bedarf}

Auf die Frage, wer bei ihnen nach der Wohnung schaue während $\mathrm{Ab}$ wesenheit wie z.B. Ferien, geben $84(37 \%)$ Nachbarn an, $75(33 \%)$ Familienangehörige, $71 \quad(31 \%)$ Freunde und Bekannte, 48 (21\%) niemanden, weil es nicht nötig sei und nur $2(0,9 \%)$ niemanden, weil niemand zur Verfügung stehe. Bei den unter-45jährigen, den Männern und den Zivilschutzpflichtigen wird dieser Dienst signifikant häufiger von Freunden und Bekannten $(42 \%, 41 \%$ resp. $43 \%)$ und signifikant seltener durch Nachbarn (24\%, $27 \%$ resp. $24 \%)$ erbracht. Bei den über-64jährigen, den Frauen und der übrigen Bevölkerung verhält es sich umgekehrt: mehr Hilfe durch Nachbarn und weniger durch Freunde und Bekannte.

Von den 84 Nachbarn, die schon nach ihrer Wohnung geschaut haben, sehen die Befragten nur 67 $(80 \%)$ regelmässig auch sonst. Also wird ein Fünftel der Nachbarn für Hilfeleistungen rekrutiert, auch wenn sie sonst keinen regelmässigen Kontakt mit den Befragten pflegen. Solche Unterschiede gibt es bei den Familienmitgliedern und Freunden nicht.

Selber besorgen die Befragten andere Wohnungen, nämlich 72 (31\%) für Nachbarn, 64 (28\%) für Familienmitglieder, 57 (25\%) für Freunde oder Bekannte und 80 (35\%) für niemanden, weil es nicht nötig ist und nur 9 (4\%) für niemanden, weil sie keine Zeit dafür haben. Solche Nachbarhilfe leisten signifikant häufiger Betagte ( $73 \%$ ) und Frauen $(67 \%)$, seltener Junge $(30 \%)$ und Männer (30\%).

Von den 72 Nachbarn, deren Wohnung sie betreuen, sehen die Befragten sonst nur $62(86 \%)$ regelmässig.

\section{Hilfe bei Krankheit und Unfall}

Von den 230 Befragten gaben 83 $(36 \%)$ an, in den letzten 5 Jahren einmal wegen Krankheit oder Unfall Hilfe benötigt $\mathrm{zu}$ haben (S.S.n.s.). Für die 77 Personen, welche die nötige Hilfe erhielten, wurde diese $\mathrm{zu} 38 \%$ von Nachbarn geleistet, (signifikant häufiger bei Frauen $(58 \%)$ und $\mathrm{Be}-$ tagten $(64 \%)$, zu $60 \%$ von $\mathrm{Fa}$ - 


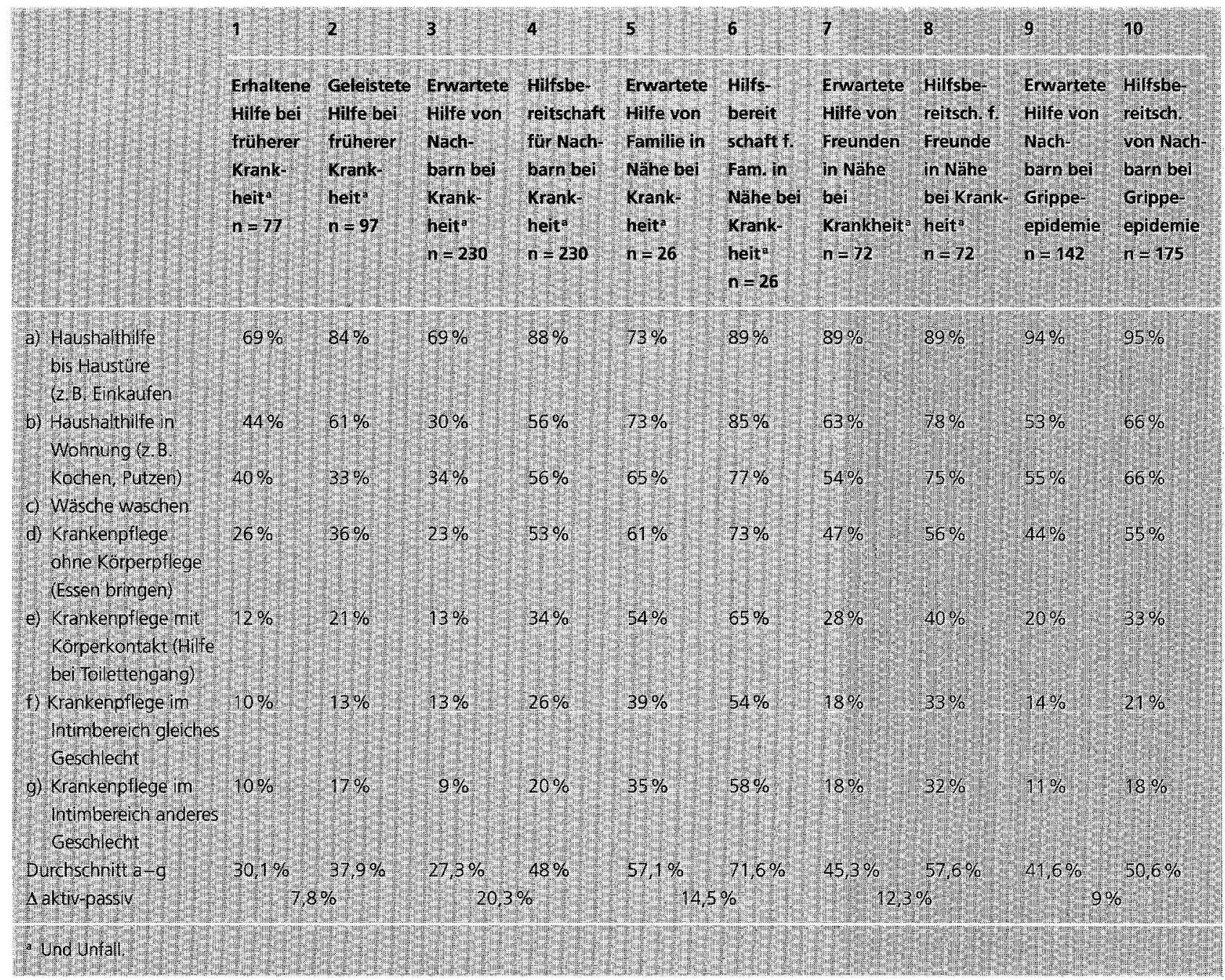

Tabelle 2. Hilfserwartung und Hilfsbereitschaft.

milienmitgliedern (S.S.n.s.), zu $36 \%$ von Freunden und Bekannten (S.S.n.s.) und zu 9\% von Spitex (S.S.n.s.). Die Art der erhaltenen Hilfe für 77 der betroffenen 83 Personen ist in Tabelle 2, Kolonne 1 dargestellt.

97 Befragte $(42 \%)$ haben in den letzten fünf Jahren jemandem wegen Krankheit oder Unfall Hilfe geleistet, 14 Personen mehr als Hilfe empfangen haben. Die aktive Hilfe war signifikant häufiger in den Subsamples übrige Bevölkerung $(54 \%)$ und Frauen $(56 \%)$ und seltener in den Subsamples Zivilschützer (34\%) und Männer (37\%), aber ohne signifikante Unterschiede bei den Altersgruppen.
Nur zwei Personen leisteten keine Hilfe weil sie keine Zeit hatten, niemand war unwillig.

Hilfe erhielten 34 Nachbarn (35\% der 97 Hilfeleistungen), 45 Familienmitglieder $(46 \%)$, und 51 $(52 \%)$ Freunde oder Bekannte. Nur 28 der 34 Nachbarn sehen die Helfenden auch sonst regelmässig $(82 \%)$, d.h. $18 \%$ der Nachbarn wird geholfen, auch wenn sonst kein regelmässiger Kontakt zu ihnen gepflegt wird. Die Art der geleisteten Hilfe ist in Tabelle 2, Kolonne 2 dargestellt.

In Tabelle 2, Kolonne 3 ist aufgeführt, welche Art von Hilfe die Befragten von Nachbarn im Falle einer Krankheit oder eines Unfalls erwarten und Kolonne 4 enthält die Hilfe, welche die Befragten für die Nachbarn zu leisten gewillt sind, die Kolonnen 5 und 6 enthalten die selben Angaben für, resp. von, in der Nähe wohnenden Familienangehörigen und die Kolonnen 7 und 8 für, resp. von, Freunden und Bekannten aus der Nachbarschaft. Im Durchschnitt sind die Befragten gewillt, $81( \pm 82)$ Minuten einzusetzen (S.S.n.s.), zu $18 \%$ eher tagsüber, zu $43 \%$ eher abends und zu $21 \%$ tagsüber und abends.

Hilfe bei Grippe

Im Fall einer Grippe mit seit drei Tagen andauernder Mühe aufzu- 


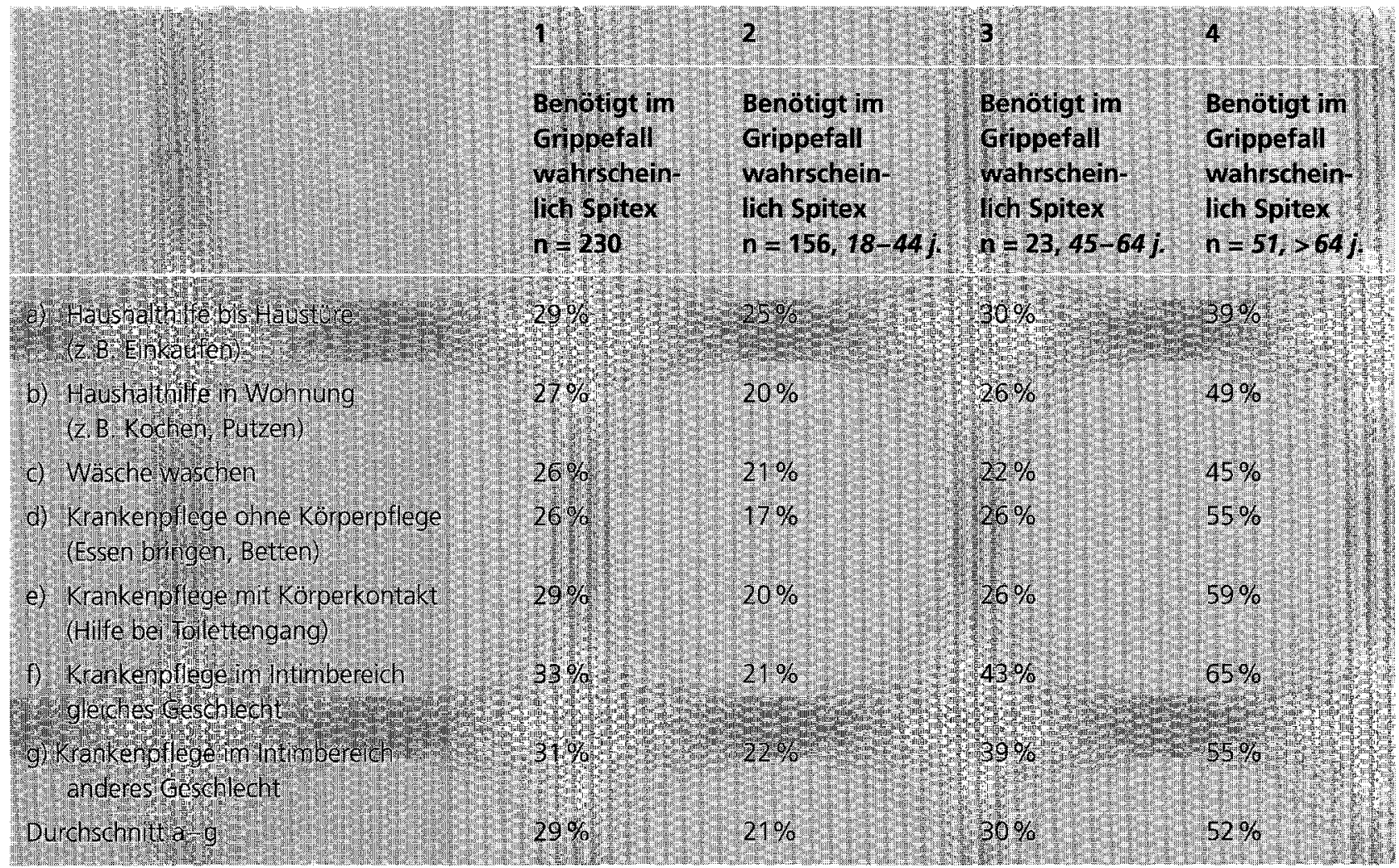

Tabelle 3. Bedarf an formeller Hilfe.

stehen (Schwindel), würden 108 Befragte $(47 \%)$ Familienangehörige, $96(42 \%)$ Freunde oder $\mathrm{Be}$ kannte, $43(19 \%)$ Nachbarn und $6(3 \%)$ andere telefonisch um Hilfe ersuchen. Bei grassierender Grippe rechnen von den $230 \mathrm{Be}$ fragten $71(31 \%)$ damit, von niemandem aus der unmittelbaren Nachbarschaft Hilfe zu bekommen (S.S.n.s.). Durchschnittlich erwarten die Befragten von 1,9 $( \pm 2,3)$ (S.S.n.s.) Nachbarn Hilfe. Von den 142, die von Nachbarn Hilfe erwarten, glauben immerhin 45 $(32 \%)$, dass mehr als zwei Nachbarn keine Hilfe leisten würden und nur $24(17 \%)$ glauben, dass alle Nachbarn helfen würden. Die Art der von Nachbarn im Grippefall erhofften Hilfe ist in Tabelle 2, Kolonne 9, dargestellt für die 142 Befragten, die eine solche Hilfe erwarten.

Bei einer Häufung von Grippefällen würden von den Befragten, wenn sie gesund sind, nur $31(14 \%)$ keinem Nachbarn helfen. Durchschnittlich sind sie bereit, $4( \pm 4,3)$ Nachbarn zu helfen, aber sie würden auch 4,5 $( \pm 13,4)$ Nachbarn sicher nicht helfen. $62(35 \%)$ würden sicher keinem Nachbarn helfen (S.S.n.s.). Die Art der Hilfe, die die dazu bereiten 175 Nachbarn $\mathrm{zu}$ leisten willens sind im Falle einer Grippeepidemie, ist in Tabelle 2, Kolonne 10 aufgeführt. 13 Befragte $(6 \%)$ kennen Personen, die niemanden haben, der ihnen helfen würde und 11 (5\%) solche, die alle Hilfe ablehnen, und zwar durchschnittlich 1,8 Personen ohne Hilfe und 1,8 Personen, die Hilfe ablehnen.

Von den Befragten glauben 206 $(90 \%)$, dass im Grippefall Familienmitglieder oder Freunde von weiter her $(>1 \mathrm{~km})$ zu Hilfe kommen würden. Nur 20 (9\%) verneinen dies (S.S.n.s.). Die Hilfe erwartenden Befragten vermuten, dass ihnen 2,2 Personen $( \pm 2)$ helfen würden. Dennoch geben $20-$ $33 \%$ der Befragten an, im Grippefall wahrscheinlich Spitexhilfe beanspruchen $\mathrm{zu}$ müssen - vor allem ältere Befragte erwarten formelle Hilfe, besonders für Pflege mit Körperkontakt und im Intimbereich (vergleiche Tabelle 3, Kolonnen 2, 3 und 4). Ausserdem erwarten sie in dieser Situation Informationen durch den amtlichen Anzeiger, das Tagblatt ( $82 \%$ ), durch Lokalradio ( $86 \%$ ) und durch Lokalfernsehen $(83 \%)$, weniger von Tages-Anzeiger $(71 \%)$ oder NZZ (52\%).

Auf die Frage, ob im Grippefall die Hilfsbereitschaft beeinflusst würde durch die Information, dass ein hohes Ansteckungsrisiko bestehe und dass es auch Todesfälle bei Jungen gebe, wurde mit einer 10stufigen Likert-Skala geantwortet $(1=$ kein Einfluss, $10=$ sehr grosser Einfluss). Dabei ergaben 


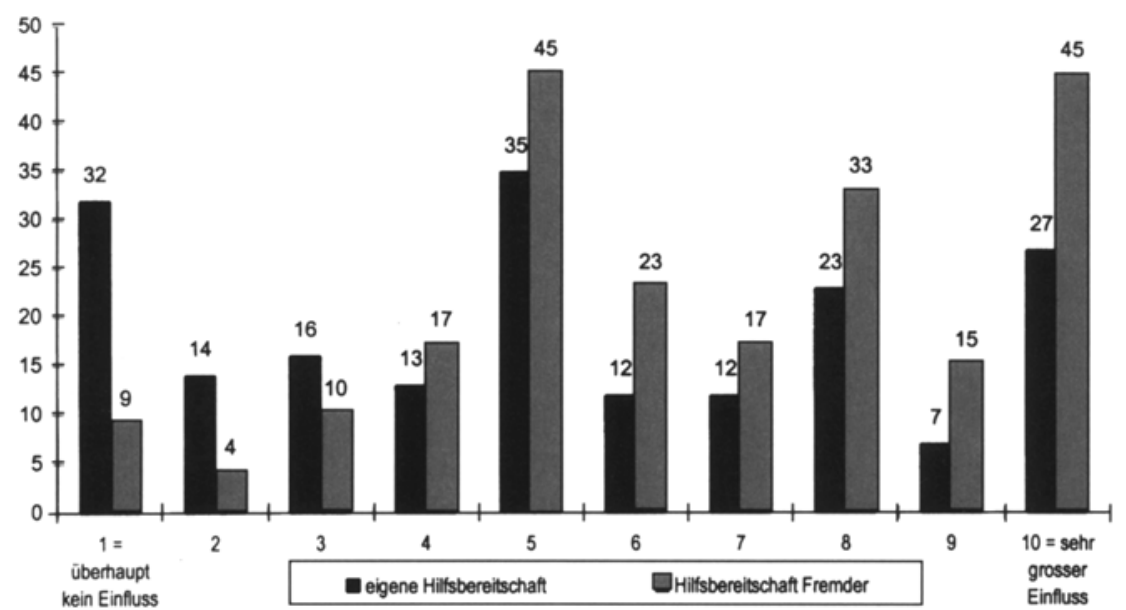

Figure 1. Welchen Einfluss haben hohe Ansteckungsgefahr und Todesfälle Jugendlicher anlässlich einer Grippepandemie auf die eigene und auf fremde Hilfsbereitschaft?

sich bei der eigenen Hilfsbereitschaft ein mittlerer Wert von 5,4 $( \pm 3,0)$ und bei derjenigen der anderen Personen ein solcher von $6,6,( \pm 2,5)$ (siehe Figur 1), wobei S.S.n.s. sind mit Ausnahme des Anteils Befragter, die mit 1 oder 10 antworten: $8 \%$ der Männer, aber $29 \%$ der Frauen; $3 \%$ der bis 44Jährigen, $30 \%$ der 45-64jährigen und sogar $41 \%$ der über 65 jährigen geben an, das hohe Ansteckungsrisiko hätte keinen Einfluss. Nur Frauen (22\%) geben wesentlich häufiger als Männer (8\%) an, dies hätte sehr grossen Einfluss, wobei sich kein signifikanter Altersunterschied fand bei den Befragten, die einen sehr grossen Einfluss angaben.

\section{Diskussion}

Dürfen die Resultate dieser Befragung für die gesamte stadtzürcherische Bevölkerung in EPH verallgemeinert werden? Von den zwei wichtigsten Kriterien der Repräsentanz, Zufallsauswahl und hohe Antwortquote, wurde nur das zweite erfüllt. Die nicht-zufällige Auswahl der Befragten führte $\mathrm{zu}$ einer deutlichen Übervertretung von Männern und Personen unter
45 Jahren, sowie von höher Gebildeten. Dies wird jedoch durch drei Fakten relativiert:

- Die Studie enthält eine Subsample-Analyse, die für die wichtigsten wahrscheinlichen Einflussvariablen auf das soziale Netz, nämlich Geschlecht, Alter und Bildungsgrad die Unterschiede analysiert, die - wenn vorhanden - anzeigen, in welche Richtung gegebenenfalls eine Verzerrung vorliegt.

- Wesentliche Aussagen der Befragungen zeigen keine signifikanten Unterschiede in den verschiedenen Subpopulationen, so dass diese Befunde wahrscheinlich ohne groben Fehler für die Gesamtpopulation verallgemeinert werden dürfen. Ein Beispiel dafür ist, dass $90 \%$ aller Betagten glauben, auch weiter entfernt lebende Familienmitglieder oder Freunde würden ihnen helfen, falls nötig.

- Einzelne Resultate sind generelle Trends, die mit unterschiedlichen Zahlen je nach Subpopulation, aber analogen Unterschieden, in jeder Population auftreten, weshalb das Pattern generalisierbar ist. Solche generalisierbare Pattern sind die
Differenzen zwischen erwarteter Hilfe und Hilfe, die Befragten zu geben bereit sind (letztere ist ca. um einen Achtel höher), oder der Trend, je näher dem Intimbereich desto geringer die erwartete und die leistungsbereite Hilfe, sowie die Aussage, dass die Mehrheit Hilfe mit Körperkontakt nur im Rahmen der Familie erwartet und zu leisten bereit ist.

Wir sind deshalb der Meinung, dass die Ergebnisse für die eingangs gestellte, praxisorientierte Frage nach Grundlagen für die Grippeplanung aussagekräftig und brauchbar sind.

Eine Stärke der vorliegenden Studie ist die in verschiedenen Bereichen hohe Konstanz der Antworten, die den Befragten sicher nicht bewusst war und deshalb die Validität der Aussagen verstärkt. Neben den oben unter Punkt 3 erwähnten generellen Pattern betrifft dies den Umstand, dass diese Pattern in allen Fragenbereichen (Kolonnen in Tabelle 2) nachweisbar und von sehr ähnlicher Grösse in jedem einzelnen Fragebereich sind. Ausserdem geben die 230 Befragten auf die Frage, ob es unter ihren Nachbarn Personen gebe, die niemanden haben oder die alle Hilfe ablehnen, 24 Personen (10,5\%) an. Umgekehrt geben von gleichviel Befragten $20(9 \%)$ an, sie hätten niemanden, der ihnen von weither zu Hilfe kommen würde.

Die Art der erhaltenen oder geleisteten Hilfe des Drittels der Befragten, die diese tatsächlich erlebt haben, entspricht weitgehend den von Nachbarn erwarteten, respektive erhaltenen Hilfe aller 230 Befragten.

Für die Validität der Studie spricht ferner der Umstand, dass die $10,5 \%$ isolierten Nachbarn, recht genau übereinstimmen mit dem Resultat der 1997 in Zürich durchgeführten repräsentativen Befragung ${ }^{2}$ von Zürcher Betagten, die in fast analoger Prozentzahl angaben, dass sie 
niemand hätten, mit dem sie sich beraten könnten.

Die scheinbaren Widersprüche der vorliegenden Resultate sind leicht erklärbar: Wenn $91 \%$ aller Befragten glauben, Familienmitglieder oder Freunde kämen ihnen in einer Notlage zu Hilfe, steht dies in scheinbarem Widerspruch zur Aussage, dass dennoch durchschnittlich $29 \%$ Spitexhilfe beanspruchen möchten, sogar $52 \%$ der befragten Betagten. Dieser Unterschied hängt sicher damit zusammen, dass viele Hemmungen haben, Hilfe von Familienmitgliedern und Freunden anzufordern, wenn Spitex zur Verfügung steht. Dies entspricht dem Umstand, dass viele - vor allem Betagte - formelle Hilfe der informellen Hilfe von Familienangehörgen vorziehen ${ }^{18}$.

Dass umgekehrt selbst von in der Nähe wohnenden Familienmitgliedern nur $35 \%$, resp. $39 \%$ Hilfe erwarten, falls sie Krankenpflege mit intimem Körperkontakt benötigen, aber nur $31 \%$, resp. $33 \%$ angeben, dafür Spitex beanspruchen zu wollen, hängt wahrscheinlich damit zusammen, dass die Mehrzahl der Befragten - wahrscheinlich zu recht - glaubt, dass im Falle einer Grippe gar keine Körperpflege mit intimem Körperkontakt nötig sein dürfte.

Der Befund, dass nur $9 \%$ der befragten Zürcher Alleinlebenden glauben, niemand käme ihnen bei einer Grippe zu Hilfe, entspricht recht genau der Antwort der 276 Befragten der SIMA VIII-Studie auf die Frage, wie leicht es für sie wäre, im Bedarfsfall jemanden für Hilfeleistungen zu gewinnen: $16 \%$ fanden, dies sei eher schwierig und $7,2 \%$, dies sei sehr schwierig. Während in der SIMA VIII-Studie bei $3 / 4$ aller Beziehungen im sozialen Netz Geben und Annehmen von Hilfe nicht zum Beziehungsinhalt gehören, ist dies in der vorliegenden Studie bei nur bei $48 \%$ der Beziehungen der Fall. In der SIMA VIII-Studie wurde jedoch nur nach effektiv geleisteter Hilfe gefragt, und $1 / 3$ der Befragen beanspruchten keinerlei Hilfe, während in unserer Studie nach der erwarteten Hilfe im Falle einer Grippeepidemie gefragt wurde. Dass für eine solche Ausnahmesituation von einem höheren Anteil der Beteiligten am sozialen Netz Hilfe erwartet wird als unter Alltagsbedingungen, war zu erwarten.

Im Gegensatz zur kulturpessimistischen Sicht der anonymen Massengesellschaft in den modernen Grossstädten, darf gemäss den Befunden dieser Studie für die grosse Anzahl Personen in EPH damit gerechnet werden, dass sie im Bedarfsfall auf Hilfe sowohl des nach wie vor starken Netzes von Primärbeziehungen als auch von Beziehungen, die auf freier Wahl beruhen, zählen können. Diese weitgehend monoplexen Beziehungen sind jedoch besonders bei älteren Personen weniger tragfähig, wenn Hilfe mit Körper- oder gar Intimkontakt nötig ist. Die Forderung nach formeller Pflegehilfe (Spitex), die $1 / 3$ der Befragten stellt, widerlegt deshalb nicht den Hauptbefund dieser und anderer Studien aus modernen Grossstädten, dass die grosse Mehrzahl auch der Alleinlebenden ein ausgedehntes und in den meisten Situationen auch für Hilfsbeziehungen tragfähiges soziales Netz aufweisen.

Für die staatlichen Massnahmeninstanzen in aussergewöhnlichen Lagen wie bei einer Grippepandemie, ist es gut zu wissen, dass praktisch die gesamte Bevölkerung über die Medien lückenlos erreicht werden kann, wenn dabei - wie heute üblich - die Kommunikation sowohl über die Print- als auch die elektronischen Medien erfolgt. Der Befund, dass auch jene $10 \%$ der stark Isolierten, die ohne hilfsbereite sozialen Beziehungen leben, auf diese Weise erreicht werden können, wird die Kommunikation der getroffenen Massnahmen und formellen Hilfsangebote gerade für diejenigen, die sie besonders nötig haben, erleichtern.

Zusammenfassend darf aus der vorliegenden Studie deshalb mit Berechtigung der Schluss gezogen werden, dass im Falle einer akuten Erkrankung wie Grippe für mindestens $10 \%$ - optimal $30 \%$ - der Bevölkerung in EPH formelle Hilfe bereitzustellen ist. Mindestens für $10 \%$, weil etwa so viele Alleinwohnende niemanden kennen, die ihnen im Grippefall helfen könnte und optimal für $30 \%$, weil soviele Alleinwohnende nur mit formeller Hilfe die notwendige Körperpflege erlangen können. 


\section{Summary}

\section{Care capacity of social networks for singles}

230 single persons living in the city of Zurich, $60 \%$ aged $18-45,10 \%$ $45-65$ and $22 \%$ older than 64 years, $70 \%$ males were subject to an enquiry. The questioned persons mostly live in woroom-apartments, on average for 11 years, they are single for 8.5 years on average. Solely $5.4 \%$ have no living relatives but $32 \%$ see them only irregularly (same for all age groups) and $4 \%$ are not persistently in touch with friends or acquaintances. $36 \%$ were needing help in the past because of sickness or accident. They got it from neighbours (38\%), friends, acquaintances $(36 \%$, family members $(17 \%)$ and from home care organisations $19 \%)$. In case of a heaw wave of influenza only $14 \%$ would not help their neighbours, they would help an average of four neighbours but also they would surely not help four neighbours. The answering persons know 23 singles $(=10 \%$ ) who oresumably would not get any help and $20 \quad 9 \%$ who would decline help. $90 \%$ of the questioned persons believe, that they would get help by family members or friends, even if those live far away, but stil $29 \%$ expect help from home care orga nisations, of the questioned over 64 years old even $52 \%$. Conclusion: $10-30 \%$ of city singles need hetp in form of home care in case of an acute iliness like heavy influenza.

H.t.

\section{Résumé}

\section{Capacité daide des résaux sociaux pour des personnes seules}

230 personnes seules, domiciliées partout dans la ville de Zurich, $60 \%$ ágées de 18 à 45 ans, $10 \%$ de $45-64$ ans et $22 \%$ plus de 65 ans, $70 \%$ masculines, font l'objet d'un questionnaire. Les personnes interrogées habitaient le plus souvent dans un appartement de deux pieces, en moyenne depuis 11 ans dans le même appartement, elles étaient seules depuis 8,5 ans en moyenne. II n'y a que $5,4 \%$ qui n'ont plus de membres de familles en vie, mais $32 \%$ ne sont pas en contact régulier avec eux (pareil pour tous les groupes d'ấge), $4 \%$ n'ont plus de contact régulier avec des amis ou des connaissances. $36 \%$ ont déjà eu besoin d'aide en raison de maladie ou d'accident, qu'a éte prodiguée par des voisins $(38 \%)$, par des amisiconnaissances $(36 \%)$, par la famille $(17 \%)$ et par l'aide communale à domicile Spitex $(9 \%)$. Dans le cas d'une épidémie de grippe II n'y a que $14 \%$ qui ne prodigueraient pas de l'aide aux voisins, en moyenne ils aideraient à quatre voisins, mais aussi ils ne rendraient súrement pas de l'aide à quatre voisins. Les personnes interrogées ont la connaissance de 23 personnes seules $(=10 \%)$ qui selon toute probabilité ne recevront pas de laide et de 20 personnes $(=9 \%)$ qui refusent de l'aide. $90 \%$ des interrogés croient que dans te cas d'une épidémie de grippe leur membres de famille ou leur amis viendraient de loin pour les aider, mais $29 \%$ des interrogées devront vraisemblablement faire recours à l'aide de Spitex, même $52 \%$ des personnes âgées plus de 64 ans. Conclusion 10-30\% des personnes seules ont besoin de l'aide de Spitex lors d'une maladie aiguè comme une grippe pénible.

\section{Literaturverzeichnis}

1 Statistisches Amt der Stadt Zürich (Hrsg) Einpersonenhaushalte. Ergebnisse der Eidgenössischen Volkszählung 1990, Zürcher Statistische Nachrichten, 3, 1994.

2 Wehrli-Schindler B. Sozialberatung für über 70jährige Personen in der Stadt Zürich - Bedürfnisse, Angebote und ihre Nutzung. Bericht des Sozialamtes der Stadt Zürich, Oktober 1997.

3 Wagner $M$, Schütze $Y$, Lang FF. Soziale Beziehungen alter Menschen. In: KU Mayer, PB Baltes (Hrsg). Die Berliner Altersstudie, Akademie Verlag Berlin, 1996: 301-319.

4 Barnes $J A$. Networks and political process. In: IC Mitchell (Hrsg). Social networks in urban situations, Manchester: University Press,1969: 51-76.

5 Mitchell JC. The concept and use of social networks. In: IC Mitchell (Hrsg). Social networks in urban situations, Manchester: University Press, 1969: 1-50.

6 Töpfer K, Stosberg M, Oswald WD. Bedingungen der Erhaltung und Förderung von Selbständigkeit im höheren Lebensalter (SIMA) - Teil VIII: Soziale Integration, soziale Netzwerke und soziale Unterstützung. Zeitschr Gerontopsych, 1998; 11:139-158.

7 Wellman $B$. The community question: The intimate networks of East Yorkers. Am J of Sociolgy, 1979; 84:1201-1231.

8 Schenk $M$. Soziale Netzwerke und Kommunikation. Tübingen: Mohr, 1984.

9 Glatzer W. Private Netzwerke als Sicherungssysteme. In: B. Seel (Hrsg), Sicherungssysteme in einer alternden Gesellschaft. Frankfurt: Campus, 1998: 80-107.

10 Bundesministerium für Familien, Senioren, Frauen und Jugend. Zweiter Bericht zur Lage der älteren Generation in der Bundesrepublik Deutschland, 1998: 186-197.

11 Fischer CS. To dwell among friends. Personal networks in town and 
city. Chicago, London: University of Chicago Press, 1982.

12 Recommendations of the $7^{\text {th }}$ European Meeting of influenza and its prevention. Europ J Epidemiol, 1994; 10:525-526.

13 Glezen $P W$. Emerging infections: Pandemic influenza. Epidemiol Review 1996; 18:64-76.

14 Dolin R, Mertens T, Hartmann A, Häcker $M$. Influenza-Virusinfektion. In: Schmalzl KJG. Harrisons Innere Medizin. Blackwell Wissenschaftsbuchhandlung. Berlin 1995, 13. Auflage: 974-979.
15 Frost WH. The epidemiology of influenza. JAMA 1991; 73:313-318.

16 Moretti-Varile T. Solitudine no ma tristesse. Vissuti ed esperienze raccontate da un gruppo di vedove. Documenti assoziazione ticinese terza età (ATTE) 2, 1996.

17 Lalive d'Epinay Ch, Maystre $C$, Bickel JF, Hagmann HM, Michel JP, Riaud JF. Un bilan de santé de la population agée. Comparaison entre deux régions de la Suisse et analyse des changements sur quinze ans (1979-1994), Cahiers médicosociaux, 1997; 41:109-131.
18 De Roulet $R$, Hirsch E, Fabre J, Rageth IP, Martin-du-Pain $R$, Posternak J.: Où logerons-nous si nous perdons notre indépendance? Schweiz. Rundschau Med (PRAXIS), 1989; 78:423-428.

\section{Korrespondenzadresse}

PD Dr. Albert Wettstein, Chefarzt Stadtärztlicher Dienst Zürich

Postfach

8035 Zürich

Fax: ++41-13 621213 